\title{
Information Filtering and Array Algorithms for Descriptor Systems Subject to Parameter Uncertainties
}

\author{
Marco Henrique Terra, Member, IEEE, João Yoshiyuki Ishihara, and Antonio Carlos Padoan, Jr.
}

\begin{abstract}
This paper presents information filters in Riccati recursions and in array algorithms for descriptor systems subject to parameters uncertainties. The filters are developed in filtered and predicted forms. The inversion of the state matrix is avoided in the new information recursive formulas. Therefore, it turns out clear that the invertibility of the state matrix, usually considered in the state-space information recursions, is not necessary. A numerical example is provided to illustrate the performance of the proposed robust array algorithms.
\end{abstract}

Index Terms-Array algorithm, descriptor systems, information filter, Kalman filter.

\section{INTRODUCTION}

$\mathbf{T}$ HE Kalman filter has been one of the most widely used tools for solving recursive estimation problems during the last 50 years. However, early after its introduction, it was noticed that the original algorithms presented some drawbacks related to practical implementation issues.

Information filtering has been considered as an alternative approach to the covariance recursions of the original Kalman filter. The filter algorithm in information form computes the inverse of the covariance matrix (the so-called information matrix), $P_{i}^{-1}$, and computes the state information estimate $P_{i}^{-1} \hat{x}_{i}$. The application of this approach is justified when, for example, there exists poor information on the initial condition $x_{0}$ of the state to be estimated. In this case, the information filter can be easily initiated with information matrix zero, whereas the covariance filter would invert very large covariance matrices. For some category of problems, the advantage of the information form over the covariance form becomes more evident. In multisensor environments, information filter is easier to distribute, initialize, and fuse than the Kalman filter [16]. It can reduce dramatically the storage and computation involved with the estimation of certain classes of large interconnected systems [2]. For more details on information filtering see, for example, [1], [14], and [16] and references therein.

On the other hand, array algorithms have been used to avoid some computational problems related to Riccati recursions. It

Manuscript received June 3, 2005; revised March 11, 2006. The associate editor coordinating the review of this manuscript and approving it for publication was Dr. Zidong Wang. This work is supported by FAPESP (Fundação de Amparo à Pesquisa do Estado de São Paulo - Brazil) under Grant 03/12574-0.

M. H. Terra and A. C. Padoan, Jr., are with the Department of Electrical Engineering, University of São Paulo at São Carlos, C.P.359, São Carlos, SP, 13566590, Brazil (e-mail: terra@sel.eesc.usp.br; carlos.padoan@ power.alstom.com).

J. Y. Ishihara is with the Department of Electrical Engineering, University of Brasilia, 70.919-970, Brasilia/DF, Brazil (e-mail: ishihara@ene.unb.br).

Digital Object Identifier 10.1109/TSP.2006.885747 is known that round-off errors can cause a loss of positive-definiteness of the computed covariance and information matrices. Fundamentally, array algorithms reduce the dynamic range in fixed-point implementations and assure better condition numbers than the conventional Kalman filter algorithm. More details on array algorithms can be found in [2], [9], [10], [14], [15], [24].

Recently, filtering and control of descriptor systems have received great attention in the literature [3]-[7], [20], [21], [25]-[28]. This interest is motivated by the fact that many systems can be modeled naturally in descriptor formulation. Applications include: economical systems [17], circuit systems [19], robotics [18], and aircraft modeling [23]. A signal processing application of descriptor filters is encountered, for example, in image restoration [8].

This paper develops information filters for descriptor systems for both nominal and robust versions. First, it is considered, Riccati equation-type formulation based on filtered and predicted estimates of [11]. In the sequel, array algorithms for the filtered and predicted information filters are derived. To the best of the authors knowledge, robust information filters and array algorithms for descriptor systems have not been addressed in the literature yet.

In the literature for usual state-space systems without uncertainties, Kalman filters in information form usually suppose the invertibility of the state matrix [matrix $F_{i}$ in the model (1)]. In this paper, nominal and robust information filters are developed without the invertibility assumption. With this, the range of applicability of information filters is enlarged. The proposed robust information singular filters, reduced to the conventional state-space systems [when $E_{i+1}$ collapses to identity in the model (1)], can be compared with the robust filter in information form of [22]. A drawback of the filter of [22] is the computation of the inverse of the information matrix at each iteration, whereas our proposed filter iterates only the information matrix (see Remark 2.4).

This paper is organized as follows. In Section II, the information estimates in filtered and predicted forms for descriptor systems, with and without uncertainties, are presented. In Section III, array algorithms for descriptor information Kalman filters are developed, and in Section IV, a numerical example illustrates the performance of the proposed algorithms.

\section{Notation}

$\Re$ is the set of real numbers, $\Re^{n}$ is the set of $n$-dimensional vectors whose elements are in $\Re, \Re^{m \times n}$ is the set of $m \times n$ real matrices, $A^{T}$ and $A^{\dagger}$ are the transpose and the pseudo-inverse 
of the matrix $A$, respectively, $P>0(P \geq 0)$ denotes that $P$ is a positive definite (semi-definite) matrix, $\|x\|$ is the Euclidian norm of $x,\|x\|_{P}$ is the weighted norm of $x$ defined by $\|x\|_{P}=$ $\left(x^{T} P x\right)^{1 / 2}$.

\section{INFORMATION FILTERS FOR DESCRIPTOR SYSTEMS}

The information filters and the array algorithms to be presented in this paper were developed to estimate the following uncertain discrete-time linear stochastic descriptor system

$$
\begin{aligned}
\left(E_{i+1}+\delta E_{i+1}\right) x_{i+1} & =\left(F_{i}+\delta F_{i}\right) x_{i}+w_{i}, i=0,1, \ldots \\
y_{i} & =\left(H_{i}+\delta H_{i}\right) x_{i}+v_{i}
\end{aligned}
$$

where $x_{i} \in \Re^{n}$ is the descriptor variable, $y_{i} \in \Re^{p}$ is the measured output, $w_{i} \in \Re^{m}$ and $v_{i} \in \Re^{p}$ are the process and measurement noises, $E_{i+1} \in \Re^{m \times n}, F_{i} \in \Re^{m \times n}$ and $H_{i} \in \Re^{p \times n}$ are the known nominal system matrices, and $\delta E_{i+1}, \delta F_{i}$ and $\delta H_{i}$ are time-varying perturbations to the nominal system matrices defined as

$$
\begin{aligned}
\delta F_{i} & =M_{f, i} \Delta_{i} N_{f, i} \\
\delta E_{i+1} & =M_{f, i} \Delta_{i} N_{e, i+1} \\
\delta H_{i} & =M_{h, i} \Delta_{i} N_{h, i} \\
\left\|\Delta_{i}\right\| & \leq 1
\end{aligned}
$$

where $M_{f, i}, M_{h, i}, N_{e, i+1}, N_{f, i}, N_{h, i}$ are known matrices and $\Delta_{i}$ is an arbitrary bounded matrix. The initial condition, the process and measurement noises, $\left\{x_{0}, w_{i}, v_{i}\right\}$, are assumed uncorrelated zero-mean random variables with second-order statistics

$$
\mathcal{E}\left(\left[\begin{array}{l}
x_{0} \\
w_{i} \\
v_{i}
\end{array}\right]\left[\begin{array}{l}
x_{0} \\
w_{j} \\
v_{j}
\end{array}\right]^{T}\right)=\left[\begin{array}{ccc}
P_{0} & 0 & 0 \\
0 & Q_{i} \delta_{i j} & 0 \\
0 & 0 & R_{i} \delta_{i j}
\end{array}\right]>0
$$

where $\delta_{i j}=1$ if $i=j$ and $\delta_{i j}=0$ otherwise.

\section{A. Nominal Estimates}

The filters for the nominal system of (1) (when $\delta F_{i}=0$, $\delta E_{i+1}=0$, and $\delta H_{i}=0$ ) solve recursively the following problems:

1) the linear least-mean-squares filtered estimate

$$
\widehat{x}_{k \mid k}=\mathcal{E}\left\{x_{k} \mid y_{k}, y_{k-1}, \ldots, y_{0}\right\}:
$$

2) the linear least-mean-squares predicted estimate

$$
\widehat{x}_{k \mid k-1}=\mathcal{E}\left\{x_{k} \mid y_{k-1}, \ldots, y_{0}\right\}
$$

The filtered estimate recursion that solves the problem 1) is given by [12], [20]

$$
\begin{aligned}
P_{i \mid i}^{-1}= & E_{i}^{T}\left(Q_{i-1}+F_{i-1} P_{i-1 \mid i-1} F_{i-1}^{T}\right)^{-1} E_{i}+H_{i}^{T} R_{i}^{-1} H_{i}(9) \\
\hat{x}_{i \mid i}= & P_{i \mid i} E_{i}^{T}\left(Q_{i}+F_{i-1} P_{i-1 \mid i-1} F_{i-1}^{T}\right)^{-1} F_{i-1} \hat{x}_{i-1 \mid i-1} \\
& +P_{i \mid i} H_{i}^{T} R_{i}^{-1} y_{i} .
\end{aligned}
$$

Remark 2.1: As it was demonstrated in [12], for the existence of a recursive solution of (9), it is required that $\left[\begin{array}{ll}E_{i}^{T} & H_{i}^{T}\end{array}\right]^{T}$ has full column rank for all $i \geq 0$. It is easy to observe that for the usual state-space systems, this condition is always satisfied.

The predicted estimation for the problem 2) is given by (11) and (12)

$$
\begin{aligned}
P_{i+1 \mid i}= & \left(E _ { i + 1 } ^ { T } \left(Q_{i}+F_{i} P_{i \mid i-1} F_{i}^{T}-F_{i} P_{i \mid i-1} H_{i}^{T}\right.\right. \\
& \left.\left.\cdot\left(R_{i}+H_{i} P_{i \mid i-1} H_{i}^{T}\right)^{-1} H_{i} P_{i \mid i-1} F_{i}^{T}\right)^{-1} E_{i+1}\right)^{-1}(11) \\
\hat{x}_{i+1 \mid i}= & P_{i+1 \mid i} E_{i+1}^{T}\left(Q_{i}+F_{i} P_{i \mid i-1} F_{i}^{T}-F_{i} P_{i \mid i-1} H_{i}^{T}\right. \\
& \left.\cdot\left(R_{i}+H_{i} P_{i \mid i-1} H_{i}^{T}\right)^{-1} H_{i} P_{i \mid i-1} F_{i}^{T}\right)^{-1} F_{i} \hat{x}_{i \mid i-1} \\
& +P_{i+1 \mid i} E_{i+1}^{T}\left(Q_{i}+F_{i} P_{i \mid i-1} F_{i}^{T}-F_{i} P_{i \mid i-1}\right. \\
& \left.\cdot H_{i}^{T}\left(R_{i}+H_{i} P_{i \mid i-1} H_{i}^{T}\right)^{-1} H_{i} P_{i \mid i-1} F_{i}^{T}\right)^{-1} \\
& \times F_{i} P_{i \mid i-1} H_{i}^{T}\left(R_{i}+H_{i} P_{i \mid i-1} H_{i}^{T}\right)^{-1}\left(y_{i}-H_{i} \hat{x}_{i \mid i-1}\right) .
\end{aligned}
$$

The existence of this predictor filter is guaranteed when $E_{i}$ has full column rank. The proof of this condition can also be seen in [12]. The filtered and predicted estimates presented above depend on the matrix $P . \mid$. . In order to express these filters in information form, where the filtering algorithms are constructed to evaluate only $P_{. \cdot .}^{-1}$, the known matrix inversion Lemma ${ }^{1}$ and some algebra are used.

1) Filtered Information Estimate: The filtered estimate in information form is given by the following equations:

$$
\begin{aligned}
P_{i \mid i}^{-1}= & E_{i}^{T} Q_{i-1}^{-1} E_{i}+H_{i}^{T} R_{i}^{-1} H_{i}-E^{T} Q_{i-1}^{-1} F_{i-1} \\
& \cdot\left(P_{i-1 \mid i-1}^{-1}+F_{i-1}^{T} Q_{i-1}^{-1} F_{i-1}\right)^{-1} F_{i-1}^{T} Q_{i-1}^{-1} E_{i} \\
P_{i \mid i}^{-1} \hat{x}_{i \mid i}= & E_{i}^{T} Q_{i}^{-1} F_{i-1}\left(F_{i-1}^{T} Q_{i}^{-1} F_{i-1}+P_{i-1 \mid i-1}^{-1}\right)^{-1} \\
& \cdot P_{i-1 \mid i-1}^{-1} \hat{x}_{i-1 \mid i-1}+H_{i}^{T} R_{i}^{-1} y_{i} .
\end{aligned}
$$

Equation (14) is obtained from (10) according to Appendix V-A. Note that (14) is a recursion for the filtered information estimate $P_{i \mid i}^{-1} \hat{x}_{i \mid i}$ which can be obtained without needing to compute $P_{i \mid i}$. Because (11) and (14) propagate the inverse of the error covariance, these equations can be used in cases where there exist no information about part or the whole initial condition $x_{0}$ (zeros in $P_{0}^{-1}$ are related with infinity values in $P_{0}$ ).

Remark 2.2: It can be noted that, even for usual state-space systems (when $E_{i}=I$ ), the main advantage of (14) if compared with the usual information filter found in the literature (cf., e.g., $[14$, Ch. 9, p. 322]), is that the invertibility of the state matrix $F_{i}$ is not necessary. This property can be verified in all other information filters developed in this paper.

2) Predicted Information Estimate: The predicted estimate recursion, (11)-(12), in information form can be written, after some algebra, as

$$
\begin{aligned}
P_{i+1 \mid i}^{-1}=E_{i+1}^{T} Q_{i}^{-1} E_{i+1}- & E_{i+1}^{T} Q_{i}^{-1} F_{i}\left(P_{i \mid i-1}^{-1}+H_{i}^{T} R_{i}^{-1} H_{i}\right. \\
& \left.+F_{i}^{T} Q_{i}^{-1} F_{i}\right)^{-1} F_{i}^{T} Q_{i}^{-1} E_{i+1}
\end{aligned}
$$

and

$$
\begin{aligned}
P_{i+1 \mid i}^{-1} & \widehat{x}_{i+1 \mid i} \\
= & E_{i+1}^{T} Q_{i}^{-1}\left(I+F_{i}\left(P_{i \mid i-1}^{-1}+H_{i}^{T} R_{i}^{-1} H_{i}\right)^{-1} F_{i}^{T} Q_{i}^{-1}\right)^{-1} \\
& \cdot F_{i}\left(P_{i \mid i-1}^{-1}+H_{i}^{T} R_{i}^{-1} H_{i}\right)^{-1} P_{i \mid i-1}^{-1} \widehat{x}_{i \mid i-1} \\
& +E_{i+1}^{T} Q_{i}^{-1}\left(I+F_{i}\left(P_{i \mid i-1}^{-1}+H_{i}^{T} R_{i}^{-1} H_{i}\right)^{-1} F_{i}^{T} Q_{i}^{-1}\right)^{-1} \\
& \cdot F_{i}\left(P_{i \mid i-1}^{-1}+H_{i}^{T} R_{i}^{-1} H_{i}\right)^{-1} H_{i}^{T} R_{i}^{-1} y_{i} .
\end{aligned}
$$

${ }^{1}(A+B D C)^{-1}=A^{-1}-A^{-1} B\left(I+D C A^{-1} B\right)^{-1} D C A^{-1}$. 
The guidelines to obtain (16) can be seen in Appendix V-B.

\section{B. Robust Information Estimates}

The robust estimates in information form of the uncertain system (1), to be presented in this section, are based on the robust singular filters given in [11]. Here, in order to simplify the filters expressions, it is assumed that $N_{e, i+1}^{T} N_{f, i}=0$. There is no loss of generality in adopting this assumption since the matrices $M_{f, i}, N_{e, i+1}$ and $N_{f, i}$ in the error modeling (2) and (3) can be always rewritten in order to satisfy this condition, as for example

$$
\begin{aligned}
M_{f, i} & =\left[\begin{array}{cc}
M_{f, i}^{11} & M_{f, i}^{12} \\
M_{f, i}^{21} & M_{f, i}^{22} \\
\ldots & \ldots \\
M_{f, i}^{m 1} & M_{f, i}^{m 2}
\end{array}\right] \\
N_{e, i+1} & =\left[\begin{array}{cccc}
N_{e, i+1}^{11} & N_{e, i+1}^{12} & \ldots & N_{e, i+1}^{1 n} \\
0 & 0 & \ldots & 0
\end{array}\right] \\
N_{f, i} & =\left[\begin{array}{cccc}
0 & 0 & \ldots & 0 \\
N_{f, i}^{21} & N_{f, i}^{22} & \ldots & N_{f, i}^{2 n}
\end{array}\right]
\end{aligned}
$$

where are $M_{f, i}^{k j}, N_{e, i+1}^{1 j}$, and $N_{f, i}^{2 j}$ are matrices of appropriate dimensions.

For the original optimal robust filtered estimates, the following sequence of robust data fitting problems are proposed: for $i=0$ solve

$$
\min _{x_{0}} \max _{\delta H_{0}}\left[\left\|x_{0}\right\|_{P_{0}^{-1}}^{2}+\left\|z_{0}-\left(H_{0}+\delta H_{0}\right) x_{0}\right\|_{R_{0}^{-1}}^{2}\right]
$$

and for $i>0$ solve (18) as shown at the bottom of the page where the uncertainties are modeled as (2)-(5).

Remark 2.3: Observe that all information filters developed in this paper are written in terms of $\left\{Q_{i}^{-1}, R_{i}^{-1}\right\}$ and $\left\{Q_{i}^{-1}, R_{i+1}^{-1}\right\}$, according to the functionals originally defined to solve these problems.

The filtered and predicted robust descriptor filters to be considered were deduced based on optimization problems defined in the following fundamental lemma [22].

Lemma 2.1: Consider the problem of solving

$$
\min _{x} \max _{\{\delta A, \delta b\}}\left[\|x\|_{Q}^{2}+\|(A+\delta A) x-(b+\delta b)\|_{W}^{2}\right]
$$

where $A$ is the data matrix, $b$ is the measurement vector which is assumed to be known, $x$ is the unknown vector, $Q=Q^{T} \geq 0$, and $W=W^{T}>0$ are given weighting matrices, $\{\delta A, \delta b\}$ are perturbations modeled by

$$
\left[\begin{array}{ll}
\delta A & \delta b
\end{array}\right]=H \Delta\left[\begin{array}{ll}
N_{a} & N_{b}
\end{array}\right],\|\Delta\| \leq 1 .
$$

The solution of the optimization problem (19) is given by

$$
\hat{x}=\left[\hat{Q}+A^{T} \hat{W} A\right]^{-1}\left[A^{T} \hat{W} b+\hat{\lambda} N_{a}^{T} N_{b}\right]
$$

where the modified weighting matrices $\{\hat{Q}, \hat{W}\}$ are defined by

$$
\begin{aligned}
\hat{Q} & :=Q+\hat{\lambda} N_{a}^{T} N_{a} ; \\
\hat{W} & :=W+W H\left(\hat{\lambda} I-H^{T} W H\right)^{\dagger} H^{T} W
\end{aligned}
$$

and $\hat{\lambda}$ is a nonnegative scalar parameter obtained by following optimization problem:

where

$$
\hat{\lambda}=\arg \min _{\lambda \geq\left\|H^{T} W H\right\|} G(\lambda)
$$

$G(\lambda):=\|x(\lambda)\|_{Q}^{2}+\lambda\left\|N_{a} x(\lambda)-N_{b}\right\|^{2}+\|A x(\lambda)-b\|_{W(\lambda)}^{2}$.

The auxiliary functions are defined by

$$
\begin{aligned}
x(\lambda) & :=\left[Q(\lambda)+A^{T} W(\lambda) A\right]^{-1}\left[A^{T} W(\lambda) b+\hat{\lambda} N_{a}^{T} N_{b}\right] \\
Q(\lambda) & :=Q+\lambda N_{a}^{T} N_{a} \\
W(\lambda) & :=W+W H\left(\lambda I-H^{T} W H\right)^{\dagger} H^{T} W .
\end{aligned}
$$

1) Robust Filtered Information Estimate: The robust singular filter based on the solutions of (17)-(18) is developed in [11]. It is not reproduced here due to space limitations. With the same arguments used to deduce the nominal information filters aforementioned, it can be shown that the robust filter for the system (1) in information form can be computed by the following algorithm.

Step 0: (Initial Conditions): If $M_{h, 0}=0$ then

$$
\begin{aligned}
P_{0 \mid 0}^{-1} & :=P_{0}^{-1}+H_{0}^{T} R_{0}^{-1} H_{0} ; \\
P_{0 \mid 0}^{-1} \hat{x}_{0 \mid 0} & :=H_{0}^{T} R_{0}^{-1} y_{0} .
\end{aligned}
$$

Otherwise determine the optimum scalar parameter $\hat{\lambda}_{-1}$ by minimizing the function $G(\lambda)$ of (24) with the identifications (67) over the interval $\lambda>\left\|M_{h, 0}^{T} R_{0}^{-1} M_{h, 0}\right\|$ and set

$$
\begin{aligned}
\hat{R}_{0}^{-1} & :=R_{0}^{-1}+R_{0}^{-1} M_{h, 0}\left(\hat{\lambda}_{-1} I-M_{h, 0}^{T} R_{0}^{-1} M_{h, 0}\right)^{-1} M_{h, 0}^{T} R_{0}^{-1} ; \\
P_{0 \mid 0}^{-1} & :=P_{0}^{-1}+H_{0}^{T} \hat{R}_{0}^{-1} H_{0}+\hat{\lambda}_{-1} N_{h, 0}^{T} N_{h, 0} ; \\
P_{0 \mid 0}^{-1} \hat{x}_{0 \mid 0} & :=H_{0}^{T} \hat{R}_{0}^{-1} y_{0} .
\end{aligned}
$$

Step 1: If $M_{f, i}=0$ and $M_{h, i+1}=0$ then $\hat{\lambda}_{i}:=0$. Otherwise determine the optimum scalar parameter $\hat{\lambda}_{i}$ by minimizing the function $G(\lambda)$ of (24) with the identifications defined in (66) (see the Appendix $V$-C) over the interval

$\hat{\lambda}_{i}>\lambda_{l, i}:=\left\|\left[\begin{array}{cc}M_{f, i}^{T} & 0 \\ 0 & M_{h, i+1}^{T}\end{array}\right]\left[\begin{array}{cc}Q_{i}^{-1} & 0 \\ 0 & R_{i+1}^{-1}\end{array}\right]\left[\begin{array}{cc}M_{f, i} & 0 \\ 0 & M_{h, i+1}\end{array}\right]\right\|$

and replace the given parameters $\left\{Q_{i}^{-1}, R_{i+1}^{-1}\right\}$ by the corrected parameters

$$
\begin{aligned}
\hat{Q}_{i}^{-1}:= & Q_{i}^{-1}+Q_{i}^{-1} M_{f, i}\left(\hat{\lambda}_{i} I-M_{f, i}^{T} Q_{i}^{-1} M_{f, i}\right)^{-1} M_{f, i}^{T} Q_{i}^{-1} ; \\
\hat{R}_{i+1}^{-1}:= & R_{i+1}^{-1}+R_{i+1}^{-1} M_{h, i+1}\left(\hat{\lambda}_{i} I-M_{h, i+1}^{T} R_{i+1}^{-1} M_{h, i+1}\right)^{-1} \\
& \times M_{h, i+1}^{T} R_{i+1}^{-1} .
\end{aligned}
$$

$\min _{\left\{x_{i}, x_{i+1}\right\}\left\{\delta E_{i+1}, \delta F_{i}, \delta H_{i+1}\right\}}\left[\left\|x_{i}-\hat{x}_{i \mid i}\right\|_{P_{i \mid i}^{-1}}^{2}+\left\|\left(E_{i+1}+\delta E_{i+1}\right) x_{i+1}-\left(F_{i}+\delta F_{i}\right) x_{i}\right\|_{Q_{i}^{-1}}^{2}+\left\|z_{i+1}-\left(H_{i+1}+\delta H_{i+1}\right) x_{i+1}\right\|_{R_{i+1}^{-1}}^{2}\right]$ 
Step 2: Update

$$
\left\{P_{i \mid i}^{-1}, P_{i \mid i}^{-1} \hat{x}_{i \mid i}\right\} \text { to }\left\{P_{i+1 \mid i+1}^{-1}, P_{i+1 \mid i+1}^{-1} \hat{x}_{i+1 \mid i+1}\right\}
$$

as (31) and (32).

$$
\begin{aligned}
P_{i+1 \mid i+1}^{-1}= & E_{i+1}^{T} \hat{Q}_{i}^{-1} E_{i+1}-E_{i+1}^{T} \hat{Q}_{i}^{-1} F_{i}\left(P_{i \mid i}^{-1}+\hat{\lambda}_{i} N_{f, i}^{T} N_{f, i}\right. \\
& \left.+F_{i}^{T} \hat{Q}_{i}^{-1} F_{i}\right)^{-1} F_{i}^{T} \hat{Q}_{i}^{-1} E_{i+1}+H_{i+1}^{T} \hat{R}_{i+1}^{-1} H_{i+1} \\
& +\hat{\lambda}_{i}\left[N_{h, i+1}^{T} N_{h, i+1}+N_{e, i+1}^{T} N_{e, i+1}\right] \\
P_{i+1 \mid i+1}^{-1} & \hat{x}_{i+1 \mid i+1} \\
= & E_{i+1}^{T} \hat{Q}_{i}^{-1}\left(I-F_{i}\left(P_{i \mid i}^{-1}+\hat{\lambda}_{i} N_{f, i}^{T} N_{f, i}\right)^{-1}\right. \\
& \left.\times F_{i}^{T} \hat{Q}_{i}^{-1}\right)^{-1} F_{i}\left(P_{i \mid i}^{-1}+\hat{\lambda}_{i} N_{f, i}^{T} N_{f, i}\right)^{-1} P_{i \mid i}^{-1} \hat{x}_{i \mid i} \\
& +H_{i+1}^{T} \hat{R}_{i+1}^{-1} y_{i+1} .
\end{aligned}
$$

One can observe that $\left[\begin{array}{c}E_{i+1} \\ H_{i+1}\end{array}\right]$ full column rank is a sufficient condition for the existence of this robust filter [11].

Remark 2.4: The robust information filter for singular systems developed in this section can be compared with the robust filter presented in [22, Table 3, p. 265]. With $E_{i}=I$, $N_{e, i+1}=0$, and $N_{h, i+1}=0$, the filter (31)-(32) reduces to the following state-space robust filter:

$$
\begin{aligned}
P_{i+1 \mid i+1}^{-1}= & \hat{Q}_{i}^{-1}-\hat{Q}_{i}^{-1} F_{i}\left(P_{i \mid i}^{-1}+\hat{\lambda}_{i} N_{f, i}^{T} N_{f, i}\right. \\
& \left.+F_{i}^{T} \hat{Q}_{i}^{-1} F_{i}\right)^{-1} F_{i}^{T} \hat{Q}_{i}^{-1}+H_{i+1}^{T} R_{i+1}^{-1} H_{i+1} \\
P_{i+1 \mid i+1}^{-1} & \hat{x}_{i+1 \mid i+1} \\
= & \hat{Q}_{i}^{-1}\left(I-F_{i}\left(P_{i \mid i}^{-1}+\hat{\lambda}_{i} N_{f, i}^{T} N_{f, i}\right)^{-1} F_{i}^{T} \hat{Q}_{i}^{-1}\right)^{-1} \\
& \times F_{i}\left(P_{i \mid i}^{-1}+\hat{\lambda}_{i} N_{f, i}^{T} N_{f, i}\right)^{-1} P_{i \mid i}^{-1} \hat{x}_{i \mid i}+H_{i+1}^{T} R_{i+1}^{-1} y_{i+1}
\end{aligned}
$$

and the state-space robust information filter of [22] is given by (the notation $E_{f, i}$ of [22] was changed to $N_{f, i}$ for easy comparison)

$$
\begin{array}{r}
P_{i+1 \mid i+1}^{-1} \hat{x}_{i+1 \mid i+1}=\left[\left(P_{i+1 \mid i+1}^{-1}-H_{i+1}^{T} \hat{R}_{i+1}^{-1} H_{i+1}\right) \hat{F}_{i} P_{i \mid i}\right] \\
\times P_{i \mid i}^{-1} \hat{x}_{i \mid i}+H_{i+1}^{T} \hat{R}_{i+1}^{-1} y_{i+1}
\end{array}
$$

where

$$
\begin{aligned}
P_{i+1 \mid i+1}^{-1} & =F_{i}^{-T} \hat{P}_{i \mid i}^{-1} F_{i}^{-1}-K_{v, i} \hat{R}_{v, i}^{-1} K_{v, i}^{T}++H_{i+1}^{T} \hat{R}_{i+1}^{-1} H_{i+1} \\
K_{v, i} & =F_{i}^{-T} \hat{P}_{i \mid i}^{-1} F_{i}^{-1} \\
R_{v, i} & =Q_{i}^{-1}+F_{i}^{-T} \hat{P}_{i \mid i}^{-1} F_{i}^{-1} \\
\hat{P}_{i \mid i}^{-1} & =P_{i \mid i}^{-1}+\hat{\lambda}_{i} N_{f, i}^{T} N_{f, i}^{T} \\
\hat{F}_{i} & =F_{i}\left(I-\hat{\lambda}_{i} \hat{P}_{i \mid i} N_{f, i}^{T} N_{f, i}^{T}\right) \\
\hat{R} & =R_{i+1}-\hat{\lambda}_{i}^{-1} H_{i+1} M_{f, i} M_{f, i}^{T} H_{i+1}^{T} .
\end{aligned}
$$

One can observe that this filter is not a genuine information filter. It is necessary to compute $P_{i \mid i}$ and $P_{i \mid i}^{-1}$ in (35).
2) Robust Predicted Information Estimate: Similar to the robust filtered estimates, to update the robust predicted estimate from $P_{i \mid i-1}^{-1} \hat{x}_{i \mid i-1}$ to $P_{i+1 \mid i}^{-1} \hat{x}_{i+1 \mid i}$, it is solved the following optimization problem for $i \geq 0$ as shown in (36) at the bottom of the page where the initial conditions are $\hat{x}_{0 \mid-1}:=\bar{x}_{0}, P_{0 \mid-1}=P_{0}$, and the uncertainties are modeled as (2)-(5). The information version of the robust predicted estimate can be computed by the following algorithm

Step 0: (Initial Conditions):

$$
\begin{aligned}
P_{0 \mid-1}^{-1} & :=P_{0}^{-1} \\
P_{0 \mid-1}^{-1} \hat{x}_{0 \mid-1} & :=P_{0}^{-1} \bar{x}_{0} .
\end{aligned}
$$

Step 1: If $M_{f, i}=0$ and $M_{h, i}=0$, then set $\hat{\lambda}_{i}=0$. Otherwise determine the optimum scalar parameter $\hat{\lambda}_{i}$ by minimizing the corresponding function $G(\lambda)$ of (24) with the identifications defined in (68) (see the appendix $V$-C) over the interval

$\hat{\lambda}_{i}>\lambda_{l, i}:=\left\|\left[\begin{array}{cc}M_{f, i}^{T} & 0 \\ 0 & M_{h, i}^{T}\end{array}\right]\left[\begin{array}{cc}Q_{i}^{-1} & 0 \\ 0 & R_{i}^{-1}\end{array}\right]\left[\begin{array}{cc}M_{f, i} & 0 \\ 0 & M_{h, i}\end{array}\right]\right\|$.

Step 2: If $\hat{\lambda}_{i} \neq 0$, replace the given parameters $\left\{Q_{i}^{-1}, R_{i}^{-1}, F_{i}\right\}$ by the corrected parameters

$$
\begin{aligned}
\mathcal{Q}_{i}^{-1} & :=\left[\begin{array}{cc}
\hat{Q}_{i}^{-1} & 0 \\
0 & I
\end{array}\right] \text { where } \hat{Q}_{i}^{-1} \text { is given by }(28) \\
\mathcal{R}_{i}^{-1} & :=\left[\begin{array}{cc}
\hat{R}_{i}^{-1} & 0 \\
0 & I
\end{array}\right] \\
\hat{R}_{i}^{-1} & :=R_{i}^{-1}+R_{i}^{-1} M_{h, i}\left(\hat{\lambda}_{i} I-M_{h, i}^{T} R_{i}^{-1} M_{h, i}\right)^{-1} M_{h, i}^{T} R_{i}^{-1} \\
\mathcal{E}_{i+1} & :=\left[\begin{array}{c}
E_{i+1} \\
\sqrt{\hat{\lambda_{i}}} N_{e, i+1}
\end{array}\right] \\
\mathcal{F}_{i} & :=\left[\begin{array}{c}
F_{i} \\
\sqrt{\hat{\lambda_{i}}} N_{f, i}
\end{array}\right] \\
\mathcal{H}_{i} & :=\left[\begin{array}{c}
H_{i} \\
\sqrt{\hat{\lambda_{i}}} N_{h, i}
\end{array}\right] .
\end{aligned}
$$

Step 3: Update

$$
\left\{P_{i \mid i-1}^{-1}, P_{i \mid i-1}^{-1} \hat{x}_{i \mid i-1}\right\} \text { to }\left\{P_{i+1 \mid i}^{-1}, P_{i+1 \mid i}^{-1} \hat{x}_{i+1 \mid i}\right\}
$$

as (41) and (42).

$$
\begin{aligned}
P_{i+1 \mid i}^{-1}= & \mathcal{E}_{i+1}^{T} \mathcal{Q}_{i}^{-1} \mathcal{E}_{i+1}-\mathcal{E}_{i+1}^{T} \mathcal{Q}_{i}^{-1} \mathcal{F}_{i}\left(P_{i \mid i-1}^{-1}\right. \\
& \left.+\mathcal{H}_{i}^{T} \mathcal{R}_{i}^{-1} \mathcal{H}_{i}+\mathcal{F}_{i}^{T} \mathcal{Q}_{i}^{-1} \mathcal{F}_{i}\right)^{-1} \mathcal{F}_{i}^{T} \mathcal{Q}_{i}^{-1} \mathcal{E}_{i+1} \\
P_{i+1 \mid i}^{-1} \widehat{x}_{i+1 \mid i} & \\
= & \mathcal{E}_{i+1}^{T} \mathcal{Q}_{i}^{-1}\left(I+\mathcal{F}_{i}\left(P_{i \mid i-1}^{-1}+\mathcal{H}_{i}^{T} \mathcal{R}_{i}^{-1} \mathcal{H}_{i}\right)^{-1}\right. \\
& \left.\cdot \mathcal{F}_{i}^{T} \mathcal{Q}_{i}^{-1}\right)^{-1} \mathcal{F}_{i}\left(P_{i \mid i-1}^{-1}+\mathcal{H}_{i}^{T} \mathcal{R}_{i}^{-1} \mathcal{H}_{i}\right)^{-1} P_{i \mid i-1}^{-1} \widehat{x}_{i \mid i-1} \\
& +\mathcal{E}_{i+1}^{T} \mathcal{Q}_{i}^{-1}\left(I+\mathcal{F}_{i}\left(P_{i \mid i-1}^{-1}+\mathcal{H}_{i}^{T} \mathcal{R}_{i}^{-1} \mathcal{H}_{i}\right)^{-1}\right. \\
& \left.\cdot \mathcal{F}_{i}^{T} \mathcal{Q}_{i}^{-1}\right)^{-1} \mathcal{F}_{i}\left(P_{i \mid i-1}^{-1}+\mathcal{H}_{i}^{T} \mathcal{R}_{i}^{-1} \mathcal{H}_{i}\right)^{-1} \mathcal{H}_{i}^{T} \mathcal{R}_{i}^{-1} y_{i}
\end{aligned}
$$

$\min _{\left\{x_{i}, x_{i+1}\right\}} \max _{\left\{\delta E_{i+1}, \delta F_{i}, \delta H_{i}\right\}}\left[\left\|x_{i}-\hat{x}_{i \mid i-1}\right\|_{P_{i \mid i-1}^{-1}}^{2}+\left\|\left(E_{i+1}+\delta E_{i+1}\right) x_{i+1}-\left(F_{i}+\delta F_{i}\right) x_{i}\right\|_{Q_{i}^{-1}}^{2}+\left\|z_{i}-\left(H_{i}+\delta H_{i}\right) x_{i}\right\|_{R_{i}^{-1}}^{2}\right]$ 


$$
\left[\begin{array}{c}
\text { Pre -Array } \\
\text { Matrix }
\end{array}\right] \overrightarrow{\text { U.T. }} \underbrace{\left[\begin{array}{c}
\text { Post }- \text { Array } \\
\text { Matrix }
\end{array}\right]}_{\text {Response matrix }}
$$

Fig. 1. Unitary transformation (U.T.) in array algorithm.

As it is detailed in [11], the existence of the filtered estimate does not assure the existence of the predicted estimate for descriptor systems, where the future dynamic has influence on the present state, then (36) was defined to solve the predicted case. For the existence of this robust predicted filter it is sufficient $E_{i+1}$ full column rank.

\section{ARRAY ALgORITHMS FOR DESCRIPTOR KALMAN FILTERS}

This section develops array algorithms to compute solutions for the Riccati equations of the information filters developed in the previous section. Array algorithm is an alternative way to solve recursive equations instead of the explicit ones. It propagates a square-root factor of a variable, which is defined for a positive semidefinite $n \times n$ matrix $P$ by a $n \times n$ matrix $A$ so that $P=A A^{T}$. These square-root factors are not unique. Defining $\Theta$ as a unitary matrix and $\Theta \Theta^{T}=\Theta^{T} \Theta=I, A \Theta$ can be defined as square-root factor of $P$. This factor can be unique if additional constraints are defined, for example if $A$ is considered triangular or Hermitian. Actually, Hermitian factor is the true square-root factor because $P=A A^{T}=A^{2}$ and $A$ can be written as $A=P^{1 / 2}$. Usually, the square-root factors of a recursive equation can be propagated by array algorithms in the following manner [14].

1) It is created a pre-array based on $i$-instant data.

2) This pre-array is transformed in a specified shape (usually triangular) using a sequence of elementary unitary transformations (rotations or reflexions).

3) The desirable values at $(i+1)$-instant can be immediately read at the post-arrays.

There is no explicit equation computation. This procedure is resumed by Fig. 1 .

The deduction of these array algorithms are based on the following Lemma.

Lemma 3.1: [14] Let $A$ and $B$ be $n \times m(n \leq m)$ matrices. Then $A A^{T}=B B^{T}$ if, and only if, there exists an $m \times m$ unitary matriz $\Theta\left(\Theta \Theta^{T}=I=\Theta^{T} \Theta\right)$ such that $A=B \Theta$.

\section{A. Filtered Information}

The filtered information estimate computes the inverse $P_{i \mid i}^{-1}$ of a Riccati recursion. In order to achieve an array algorithm that propagates the square-root factor $P_{i \mid i}^{-1 / 2}$, the right side of (11) is written as Schur complement ${ }^{2}$ of $\left\{P_{i-1 \mid i-1}^{-1}+F_{i-1}^{T} Q_{i-1}^{-1} F_{i-1}\right\}$ in

$$
\left[\begin{array}{rc}
P_{i-1 \mid i-1}^{-1}+F_{i-1}^{T} Q_{i-1}^{-1} F_{i-1} & F_{i-1}^{T} Q_{i-1}^{-1} E_{i} \\
E_{i}^{T} Q_{i-1}^{-1} F_{i-1} & E_{i}^{T} Q_{i-1}^{-1} E_{i}+H_{i}^{T} R_{i}^{-1} H_{i}
\end{array}\right] .
$$

${ }^{2}$ Schur complement of $A$ in $M=\left[\begin{array}{ll}A & B \\ C & D\end{array}\right]$ is given by $\Delta_{A} \equiv D-$ $C A^{-1} B$
According to Lemma 3.1, one must find an equality $A A^{T}=$ $B B^{T}$. Then, first, (43) is factorized as $A_{f, i} A_{f, i}^{T}$ where

$$
A_{f, i}=\left[\begin{array}{ccc}
P_{i-1 \mid i-1}^{-1 / 2} & F_{i-1}^{T} Q_{i-1}^{-1 / 2} & 0 \\
0 & E_{i}^{T} Q_{i-1}^{-1 / 2} & H_{i}^{T} R_{i}^{-1 / 2}
\end{array}\right] .
$$

Using other property of Schur complement ${ }^{3}$, (43) can be written as

$$
\begin{gathered}
{\left[\begin{array}{cc}
I & 0 \\
E_{i}^{T} Q_{i-1}^{-1} F_{i-1} A_{i-1}^{-1} & I
\end{array}\right]\left[\begin{array}{cc}
A_{i-1}^{1 / 2} & 0 \\
0 & P_{i \mid i}^{-1 / 2}
\end{array}\right]\left[\begin{array}{cc}
A_{i-1}^{1 / 2} & 0 \\
0 & P_{i \mid i}^{-1 / 2}
\end{array}\right]} \\
\cdot\left[\begin{array}{cc}
I & A_{i-1}^{-1} F_{i-1}^{T} Q_{i-1}^{-1} E_{i} \\
0 & I
\end{array}\right]
\end{gathered}
$$

where $A_{i-1} \equiv P_{i-1 \mid i-1}^{-1}+F_{i-1}^{T} Q_{i-1}^{-1} F_{i-1}$ is Hermitian and positive-definite. With (46), (43) can be factorized as $B_{f, i} B_{f, i}^{T}$ where

$$
B_{f, i}=\left[\begin{array}{ccc}
A_{i-1}^{1 / 2} & 0 & 0 \\
E_{i}^{T} Q_{i-1}^{-1} F_{i-1} A_{i-1}^{-1 / 2} & P_{i \mid i}^{-1 / 2} & 0
\end{array}\right] .
$$

The array algorithm for the filtered estimate, in information form, is explicitly established via (47), (44), and a unitary matrix $\Theta$, as

$$
\begin{aligned}
{\left[\begin{array}{ccc}
P_{i-1 \mid i-1}^{-1 / 2} & F_{i-1}^{T} Q_{i-1}^{-1 / 2} & 0 \\
0 & E_{i}^{T} Q_{i-1}^{-1 / 2} & H_{i}^{T} R_{i}^{-1 / 2}
\end{array}\right] \Theta } & \\
& =\left[\begin{array}{ccc}
A_{i-1}^{1 / 2} & 0 & 0 \\
E_{i}^{T} Q_{i-1}^{-1} F_{i-1} A_{i-1}^{-1 / 2} & P_{i \mid i}^{-1 / 2} & 0
\end{array}\right]
\end{aligned}
$$

\section{B. Predicted Information}

Following the procedure used to find the array algorithm for the information filtered estimate, the predicted information estimate computed by (15) is rewritten as Schur complement of $\left\{P_{i \mid i-1}^{-1}+H_{i}^{T} R_{i}^{-1} H_{i}+F_{i}^{T} Q_{i}^{-1} F_{i}\right\}$ in

$$
\left[\begin{array}{cc}
P_{i \mid i-1}^{-1}+H_{i}^{T} R_{i}^{-1} H_{i}+F_{i}^{T} Q_{i}^{-1} F_{i} & F_{i}^{T} Q_{i}^{-1} E_{i+1} \\
E_{i+1}^{T} Q_{i}^{-1} F_{i} & E_{i+1}^{T} Q_{i}^{-1} E_{i+1}
\end{array}\right] .
$$

Equation (49) is factorized as $A_{p, i} A_{p, i}^{T}$ where

$$
A_{p, i}=\left[\begin{array}{ccc}
P_{i \mid i-1}^{-1 / 2} & F^{T} Q_{i}^{-1 / 2} & H^{T} R^{-1 / 2} \\
0 & E^{T} Q_{i}^{-1 / 2} & 0
\end{array}\right] .
$$

Following the same decomposition defined in the footnote (45), (49) can be factorized as $B_{p, i} B_{p, i}^{T}$, where

$$
B_{p, i}=\left[\begin{array}{ccc}
X_{i} & 0 & 0 \\
Y_{i} & P_{i+1 \mid i}^{-1 / 2} & 0
\end{array}\right]
$$

${ }^{3}$ A block matrix $\left[\begin{array}{ll}A & B \\ C & D\end{array}\right]$ can be factorized as

$$
\left[\begin{array}{cc}
A & B \\
C & D
\end{array}\right]=\left[\begin{array}{cc}
I & 0 \\
C A^{-1} & I
\end{array}\right]\left[\begin{array}{cc}
A & 0 \\
0 & \Delta_{A}
\end{array}\right]\left[\begin{array}{cc}
I & A^{-1} B \\
0 & I
\end{array}\right] .
$$


with $X_{i}=\left(P_{i \mid i-1}^{-1}+H_{i}^{T} R_{i}^{-1} H_{i}+F_{i}^{T} Q_{i}^{-1} F_{i}\right)^{1 / 2}$ and $Y_{i}=E_{i+1}^{T} Q_{i}^{-1} F_{i}\left(P_{i \mid i-1}^{-1}+H_{i}^{T} R_{i}^{-1} H_{i}+F_{i}^{T} Q_{i}^{-1} F_{i}\right)^{-1 / 2}$. Then, there exists a unitary matrix $\Theta$ such that the array algorithm for the information predicted form can be computed as (52) as shown at the bottom of the page.

\section{Robust Filtered Information}

Considering $C_{i}=N_{h, i+1}^{T} N_{h, i+1}+N_{e, i+1}^{T} N_{e, i+1}$, the right side of (31) can be written as the Schur complement of $K_{i}=$ $P_{i \mid i}^{-1}+\hat{\lambda}_{i}^{1 / 2} N_{f, i}^{T} N_{f, i} \hat{\lambda}_{i}^{1 / 2}+F_{i}^{T} \hat{Q}_{i}^{-1} F_{i}$ in

$$
\left[\begin{array}{cc}
K_{i} & F_{i}^{T} \hat{Q}_{i}^{-1} E_{i+1} \\
E_{i+1}^{T} \hat{Q}_{i}^{-1} F_{i} & H_{i+1}^{T} \hat{R}_{i+1}^{-1} H_{i+1}+\hat{\lambda}_{i}^{1 / 2} C_{i} \hat{\lambda}_{i}^{1 / 2}
\end{array}\right]
$$

that can be factorized as $A_{r f, i} A_{r f, i}^{T}$, where $A_{r f, i}$ is defined in (54) as shown at the bottom of the page. (52) can be also rewritten as

$\left[\begin{array}{cc}I & 0 \\ E_{i+1}^{T} \hat{Q}_{i}^{-1} F_{i} K_{i}^{-1} & I\end{array}\right]\left[\begin{array}{cc}K_{i} & 0 \\ 0 & P_{i+1 \mid i+1}^{-1}\end{array}\right]\left[\begin{array}{cc}I & K_{i}^{-1} F_{i}^{T} \hat{Q}_{i}^{-1} E_{i+1} \\ 0 & I\end{array}\right]$

and

$$
\begin{aligned}
& {\left[\begin{array}{ccc}
K_{i}^{1 / 2} & 0 & 0 \\
E_{i+1}^{T} \hat{Q}_{i}^{-1} F_{i} K_{i}^{-1 / 2} & P_{i+1 \mid i+1}^{-1 / 2} & 0
\end{array}\right] } \\
& \cdot\left[\begin{array}{ccc}
K_{i}^{1 / 2} & 0 & 0 \\
E_{i+1}^{T} \hat{Q}_{i}^{-1} F_{i} K_{i}^{-1 / 2} & P_{i+1 \mid i+1}^{-1 / 2} & 0
\end{array}\right]^{T}
\end{aligned}
$$

Therefore, based on Lemma 3.1, the array algorithm for robust filtered estimate of singular systems, in information form, is given by (57) as shown at the bottom of the page.

\section{Robust Predicted Information}

Following the line adopted to deduce the array algorithm for the nominal predicted estimate, and observing the similarities between (15) and (41), the array algorithm for the robust predicted estimate is given by (58) as shown at the bottom of the page.

\section{NUMERICAL EXAMPLE}

A numerical example was performed in order to compare the descriptor information filters, in nominal and in robust forms (13) and (31), with their array versions (48) and (57), respectively. The singular values of $P_{i \mid i}^{-1}$ $\left(\sigma_{j}\left(P_{i \mid i}^{-1}\right): \quad j=1,2, \ldots, n\right)$ were computed first for the Riccati equations via Matlab double precision floating-point processing (this floating-point processing was used as reference, for comparison), and second for the Riccati equations and array algorithms were computed via Matlab (Simulink toolbox) 16-bit fixed-point architecture. The nominal model of (1) is considered as

$$
\begin{aligned}
E_{i} & =\left[\begin{array}{ccc}
1.14 & 0 & 0 \\
0 & 1.17 & 0 \\
0 & 0 & 0
\end{array}\right], F_{i}=\left[\begin{array}{ccc}
0.97 & 0 & 0 \\
0.27 & -0.78 & 0 \\
0.12 & 0.12 & 0.67
\end{array}\right], \\
H_{i} & =\left[\begin{array}{ccc}
0.11 & 0 & 0 \\
0 & 0.52 & 0 \\
0 & 0 & 0.31
\end{array}\right], Q_{i}^{-1}=\left[\begin{array}{ccc}
7.70 & 0 & 0 \\
0 & 5.56 & 0 \\
0 & 0 & 7.14
\end{array}\right], \\
R_{i}^{-1} & =\left[\begin{array}{ccc}
12.50 & 0 & 0 \\
0 & 33.33 & 0 \\
0 & 0 & 50
\end{array}\right],
\end{aligned}
$$

$$
\left[\begin{array}{ccc}
P_{i \mid i-1}^{-1 / 2} & F_{i}^{T} Q_{i}^{-1 / 2} & H_{i}^{T} R_{i}^{-1 / 2} \\
0 & E_{i+1}^{T} Q_{i}^{-1 / 2} & 0
\end{array}\right] \Theta=\left[\begin{array}{ccc}
\left(P_{i \mid i-1}^{-1}+H_{i}^{T} R_{i}^{-1} H_{i}+F_{i}^{T} Q_{i}^{-1} F_{i}\right)^{1 / 2} & 0 & 0 \\
E_{i+1}^{T} Q_{i}^{-1} F_{i}\left(P_{i \mid i-1}^{-1}+H_{i}^{T} R_{i}^{-1} H_{i}+F_{i}^{T} Q_{i}^{-1} F_{i}\right)^{-1 / 2} & P_{i+1 \mid i}^{-1 / 2} & 0
\end{array}\right]
$$

$$
A_{r f, i}=\left[\begin{array}{cccccc}
P_{i \mid i}^{-1 / 2} & F_{i}^{T} \hat{Q}_{i}^{-1 / 2} & \hat{\lambda}_{i}^{1 / 2} N_{f, i}^{T} & 0 & 0 & 0 \\
0 & E_{i+1}^{T} \hat{Q}_{i}^{-1 / 2} & 0 & H_{i+1}^{T} \hat{R}_{i+1}^{-1 / 2} & \hat{\lambda}_{i}^{1 / 2} N_{h, i+1}^{T} & \hat{\lambda}_{i}^{1 / 2} N_{e, i+1}^{T}
\end{array}\right]
$$

$$
\begin{aligned}
& {\left[\begin{array}{cccccc}
P_{i \mid i}^{-1 / 2} & F_{i}^{T} \hat{Q}_{i}^{-1 / 2} & \hat{\lambda}_{i}^{1 / 2} N_{f, i}^{T} & 0 & 0 & 0 \\
0 & E_{i+1}^{T} \hat{Q}_{i}^{-1 / 2} & 0 & H_{i+1}^{T} \hat{R}_{i+1}^{-1 / 2} & \hat{\lambda}_{i}^{1 / 2} N_{h, i+1}^{T} & \hat{\lambda}_{i}^{1 / 2} N_{e, i+1}^{T}
\end{array}\right] \Theta} \\
& =\left[\begin{array}{cccccc}
K_{i}^{1 / 2} & 0 & 0 & 0 & 0 & 0 \\
E_{i+1}^{T} \hat{Q}_{i}^{-1} F_{i} K_{i}^{-1 / 2} & P_{i+1 \mid i+1}^{-1 / 2} & 0 & 0 & 0 & 0
\end{array}\right] .
\end{aligned}
$$

$$
\left[\begin{array}{ccc}
P_{i \mid i-1}^{-1 / 2} & \mathcal{H}_{i}^{T} \mathcal{R}_{i}^{-1 / 2} & \mathcal{F}_{i}^{T} \mathcal{Q}_{i}^{-1 / 2} \\
0 & 0 & \mathcal{E}_{i+1}^{T} \mathcal{Q}_{i}^{-1 / 2}
\end{array}\right] \Theta=\left[\begin{array}{ccc}
\left(P_{i \mid i-1}^{-1}+\mathcal{H}_{i}^{T} \mathcal{R}_{i}^{-1} \mathcal{H}_{i}+\mathcal{F}_{i}^{T} \mathcal{Q}_{i}^{-1} \mathcal{F}_{i}\right)^{1 / 2} & 0 & 0 \\
\mathcal{E}_{i+1}^{T} \mathcal{Q}_{i}^{-1} \mathcal{F}_{i}\left(P_{i \mid i-1}^{-1}+\mathcal{H}_{i}^{T} \mathcal{R}_{i}^{-1} \mathcal{H}_{i}+\mathcal{F}_{i}^{T} \mathcal{Q}_{i}^{-1} \mathcal{F}_{i}\right)^{-1 / 2} & P_{i+1 \mid i}^{-1 / 2} & 0
\end{array}\right]
$$


TABLE I

MSE BETWEen Fixed-Point AND FloATING-Point IMPLEMENTATIONS OF $\sigma_{j}\left(P_{i \mid i}^{-1}\right)$

\begin{tabular}{|r|c||c|c|c|}
\hline Type & Implementation & $M S E_{1}$ & $M S E_{2}$ & $M S E_{3}$ \\
\hline \hline \multirow{2}{*}{$\begin{array}{c}\text { Nominal } \\
\left(\times 10^{-5}\right)\end{array}$} & Riccati & 22.9510 & 67.1321 & 0.0026 \\
\cline { 2 - 5 } & Array Algorithm & 0.3410 & 0.0033 & 0.0007 \\
\hline \hline Robust & Riccati & 68.6992 & 3.8622 & 1.4211 \\
\cline { 2 - 5 } & Array Algorithm & 0.0044 & 0.0003 & 0.0004 \\
\hline
\end{tabular}

and the uncertainties of (1) as

$$
\begin{aligned}
& N_{e}=\left[\begin{array}{ccc}
0.1 & 0 & 0 \\
0 & 0 & 0 \\
0 & 0 & 0
\end{array}\right], \quad N_{f}=\left[\begin{array}{ccc}
0 & 0 & 0 \\
0 & 0.05 & 0 \\
0 & 0 & 0.3
\end{array}\right], \\
& N_{h}=\left[\begin{array}{ccc}
0.002 & 0 & 0 \\
0 & 0.03 & 0 \\
0 & 0 & 0.002
\end{array}\right], \\
& M_{f}=\left[\begin{array}{ccc}
0.5 & 0 & 0 \\
0 & 0.5 & 0 \\
0 & 0 & 1.3
\end{array}\right], M_{h}=\left[\begin{array}{ccc}
8 & 0 & 0 \\
0 & .8 & 0 \\
0 & 0 & .8
\end{array}\right] .
\end{aligned}
$$

Table I shows the mean square error (mse) between the fixed-point and floating-point implementations, computed via singular values of $P_{i \mid i}^{-1}$, defined as

$$
\text { mse }_{j}=\frac{1}{T} \sum_{i=1}^{T}\left(\sigma_{j}\left(P_{i \mid i}^{-1}\right)_{f l}-\sigma_{j}\left(P_{i \mid i}^{-1}\right)_{f i}\right)^{2}
$$

where the Riccati equations and array algorithms are computed for the nominal model with parameters (59) and for the model with uncertainties (60); $T$ is the number of iterations. When the floating-point configuration is used to compute $P_{i \mid i}^{-1}$, the results obtained through array algorithm and Riccati equation are almost the same, as it was expected. One can observe in Table I the advantage of array algorithms for nominal and robust descriptor filters. The adjust of the robust filter, (31) and (57), was performed as $\hat{\lambda}_{i}=40$ for all $i$. Fig. 2 displays the singular values of $P_{i \mid i}^{-1}$ for robust descriptor filtering in information form for three different implementations: Riccati equation computed through the floating-point processing; Array algorithm and Riccati equation computed via fixed-point processing. One can observe that the array algorithm implemented with a fixed-point configuration has produced the same results obtained when floating-point configuration is used, the singular values of $P_{i \mid i}^{-1}$ are almost the same. There exist expressive differences between the singular values when $P_{i \mid i}^{-1}$ is computed via Riccati equation and with a fixed-point configuration.

\section{CONCLUSION}

This paper has developed Kalman-type recursive estimates of descriptor systems in filtered information and predicted information versions for nominal and robust estimation problems. The respective array algorithms have also been presented. These new information filters do not require the invertibility of the matrix $F_{i}$ for four important filtering classes: of robust descriptor systems, of robust state-space systems, and of the respective

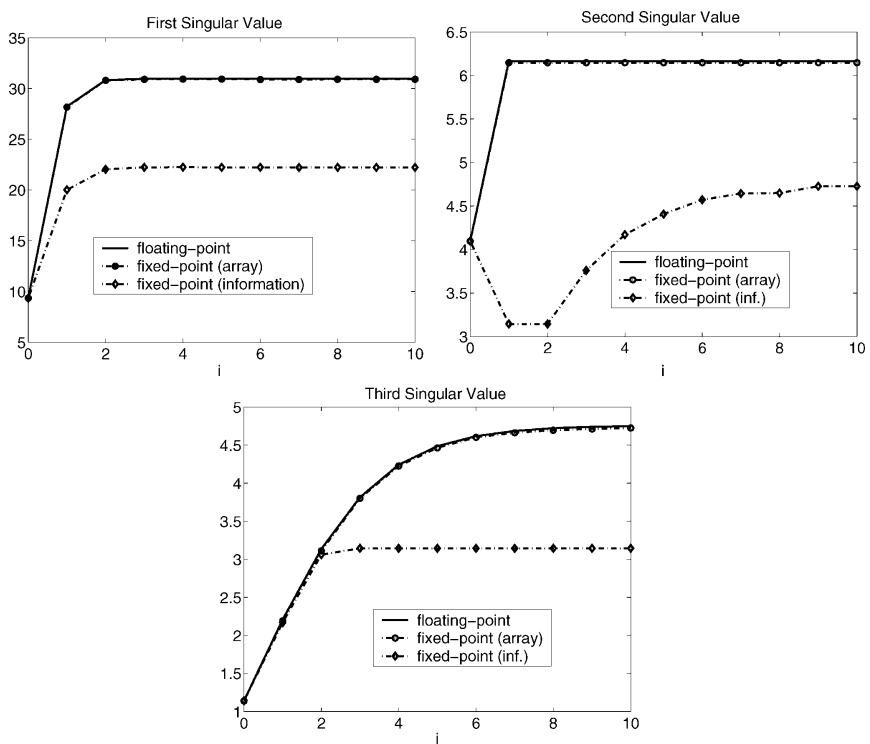

Fig. 2. Singular values of $P_{i \mid i}^{-1}$ for robust descriptor filtering, in information form, for three different implementations.

nominal descriptor and state-space systems. The proposed robust information filters and array algorithms, are the first filters developed in the literature for uncertain descriptor systems and also for uncertain state-space systems. The only robust information filter that was found, presented in [22], is not exactly a robust information filter. The numerical example shows that in fixed-point implementations, the information matrices obtained by array algorithm are closer to the correct values (the results obtained via floating-point are assumed as reference) than those obtained by Kalman-type implementation.

\section{APPENDIX A \\ NOMINAL FILTERED INFORMATION FILTER}

Equation (14) is obtained after the following algebra: first it is applied the matrix inversion Lemma in (10) and $P_{i \mid i}^{-1}$ is multiplied in both sides of the equality

$$
\begin{aligned}
P_{i \mid i}^{-1} \hat{x}_{i \mid i}=E_{i}^{T} & \left(Q_{i}^{-1}-Q_{i}^{-1} F_{i-1}\left(P_{i-1 \mid i-1}^{-1}+F_{i-1}^{T} Q_{i}^{-1}\right.\right. \\
& \left.\left.\times F_{i-1}\right)^{-1} F_{i-1}^{T} Q_{i}^{-1}\right) F_{i-1} \hat{x}_{i-1 \mid i-1}+H_{i}^{T} R_{i}^{-1} y_{i}
\end{aligned}
$$

and then $E_{i}^{T} Q_{i}^{-1} F_{i-1}\left(P_{i-1 \mid i-1}^{-1}+F_{i-1}^{T} Q_{i}^{-1} F_{i-1}\right)^{-1}$ is put in evidence.

\section{APPENDIX B}

\section{NOMINAL PREDICTED INFORMATION FILTER}

To obtain (16), the matrix inversion Lemma is applied in (12) and $P_{i \mid i}^{-1}$ is multiplied in both sides of the equality

$$
\begin{aligned}
P_{i+1 \mid i}^{-1} \hat{x}_{i+1 \mid i} & \\
= & E_{i+1}^{T}\left(Q_{i}+F_{i}\left(P_{i \mid i-1}^{-1}+H_{i}^{T} R_{i}^{-1} H_{i}\right)^{-1} F_{i}^{T}\right)^{-1} \\
& \cdot F_{i} \hat{x}_{i \mid i-1}+E_{i+1}^{T}\left(Q_{i}+F_{i}\left(P_{i \mid i-1}^{-1}+H_{i}^{T} R_{i}^{-1} H_{i}\right) F_{i}^{T}\right)^{-1} \\
& \cdot F_{i} P_{i \mid i-1} H_{i}^{T}\left(R_{i}+H_{i} P_{i \mid i-1} H_{i}^{T}\right)^{-1}\left(y_{i}-H_{i} \hat{x}_{i \mid i-1}\right) .
\end{aligned}
$$


This equation can be rewritten as

$$
\begin{aligned}
& P_{i+1 \mid i}^{-1} \widehat{x}_{i+1 \mid i} \\
&=E_{i+1}^{T}\left(Q_{i}+F_{i}\left(P_{i \mid i-1}^{-1}+H_{i}^{T} R_{i}^{-1} H_{i}\right)^{-1} F_{i}^{T}\right)^{-1} \\
& \quad \times\left(F _ { i } \left(P_{i \mid i-1} P_{i \mid i-1}^{-1}-P_{i \mid i-1} H_{i}^{T}\left(R_{i}+H_{i} P_{i \mid i-1} H_{i}^{T}\right)^{-1}\right.\right. \\
&\left.\left.\cdot H_{i} P_{i \mid i-1} P_{i \mid i-1}^{-1}\right) \widehat{x}_{i \mid i-1}+F_{i} P_{i \mid i-1} H_{i}^{T}\left(R_{i}+H_{i} P_{i \mid i-1} H_{i}^{T}\right)^{-1} y_{i}\right) .
\end{aligned}
$$

Using again the matrix inversion Lemma and multiplying the last term of (63) by $\left(P_{i \mid i-1}^{-1}+H_{i}^{T} R_{i}^{-1} H_{i}\right)^{-1}\left(P_{i \mid i-1}^{-1}+\right.$ $\left.H_{i}^{T} R_{i}^{-1} H_{i}\right)$, one obtains

$$
\begin{aligned}
& P_{i+1 \mid i}^{-1} \widehat{x}_{i+1 \mid i} \\
&= E_{i+1}^{T}\left(Q_{i}+F_{i}\left(P_{i \mid i-1}^{-1}+H_{i}^{T} R_{i}^{-1} H_{i}\right)^{-1} F_{i}^{T}\right)^{-1} \\
& \cdot F_{i}\left(\left(P_{i \mid i-1}^{-1}+H_{i}^{T} R_{i}^{-1} H_{i}\right)^{-1}\right. \\
& \times P_{i \mid i-1}^{-1} \widehat{x}_{i \mid i-1}+\left(P_{i \mid i-1}^{-1}+H_{i}^{T} R_{i}^{-1} H_{i}\right)^{-1} \\
&\left.\cdot\left(P_{i \mid i-1}^{-1}+H_{i}^{T} R_{i}^{-1} H_{i}\right) P_{i \mid i-1} H_{i}^{T}\left(R_{i}+H_{i} P_{i \mid i-1} H_{i}^{T}\right)^{-1} y_{i}\right)
\end{aligned}
$$

putting in evidence $\left(P_{i \mid i-1}^{-1}+H_{i}^{T} R_{i}^{-1} H_{i}\right)^{-1}$, (64) can be rewritten as

$$
\begin{aligned}
P_{i+1 \mid i}^{-1} \widehat{x}_{i+1 \mid i} & \\
= & E_{i+1}^{T}\left(Q_{i}+F_{i}\left(P_{i \mid i-1}^{-1}+H_{i}^{T} R_{i}^{-1} H_{i}\right)^{-1} F_{i}^{T}\right)^{-1} \\
& \cdot F_{i}\left(P_{i \mid i-1}^{-1}+H_{i}^{T} R_{i}^{-1} H_{i}\right)^{-1}\left(P_{i \mid i-1}^{-1} \widehat{x}_{i \mid i-1}\right. \\
& \left.+\left(H_{i}^{T}+H_{i}^{T} R_{i}^{-1} H_{i} P_{i \mid i-1} H_{i}^{T}\right)\left(R_{i}+H_{i} P_{i \mid i-1} H_{i}^{T}\right)^{-1} y_{i}\right)
\end{aligned}
$$

and then, withdrawing $Q_{i}$ of the inverse and rewriting $\left(H_{i}^{T}+\right.$ $\left.H_{i}^{T} R_{i}^{-1} H_{i} P_{i \mid i-1} H_{i}^{T}\right)$ as $H_{i}^{T} R_{i}^{-1}\left(R_{i}+H_{i} P_{i \mid i-1} H_{i}^{T}\right)$ one obtains (16).

\section{APPENDIX C \\ ROBUST INFORMATION FILTERS}

To compute the optimal robust filtered estimates, the following identifications are required in Lemma 2.1 to find $\hat{\lambda}_{i}$

$$
\begin{aligned}
A & \leftarrow\left[\begin{array}{cc}
-F_{i} & E_{i+1} \\
0 & H_{i+1}
\end{array}\right] ; \quad b \leftarrow\left[\begin{array}{c}
F_{i} \hat{x}_{i \mid i} \\
z_{i+1}
\end{array}\right] \\
\delta A & \leftarrow\left[\begin{array}{cc}
-\delta F_{i} & \delta E_{i+1} \\
0 & \delta H_{i+1}
\end{array}\right] ; \delta b \leftarrow\left[\begin{array}{c}
\delta F_{i} \hat{x}_{i \mid i} \\
0
\end{array}\right] \\
Q & \leftarrow\left[\begin{array}{cc}
P_{i \mid i}^{-1} & 0 \\
0 & 0
\end{array}\right] ; W \leftarrow\left[\begin{array}{cc}
Q_{i}^{-1} & 0 \\
0 & R_{i+1}^{-1}
\end{array}\right] \\
N_{a} & \leftarrow\left[\begin{array}{cc}
-N_{f, i} & N_{e, i+1} \\
0 & N_{h, i+1}
\end{array}\right] ; N_{b} \leftarrow\left[\begin{array}{c}
N_{f, i} \hat{x}_{i \mid i} \\
0
\end{array}\right] \\
H & \leftarrow\left[\begin{array}{cc}
M_{f, i} & 0 \\
0 & M_{h, i}
\end{array}\right]
\end{aligned}
$$

and for the initial condition, the following identifications are considered:

$$
\begin{aligned}
& A \leftarrow H_{0} ; \quad b \leftarrow z_{0} ; \delta A \leftarrow \delta H_{0} \\
& \delta b \leftarrow 0 ; \quad Q \leftarrow P_{0}^{-1} ; W \leftarrow R_{0}^{-1} \\
& H \leftarrow M_{h, 0} ; \quad N_{a} \leftarrow N_{h, 0} ; \quad N_{b} \leftarrow 0 .
\end{aligned}
$$

To compute the optimal robust predicted estimates, the following identifications are required in Lemma 2.1 to find $\hat{\lambda}_{i}$ :

$$
\begin{aligned}
A & \leftarrow\left[\begin{array}{cc}
-F_{i} & E_{i+1} \\
H_{i} & 0
\end{array}\right] ; b \leftarrow\left[\begin{array}{c}
F_{i} \hat{x}_{i \mid i-1} \\
z_{i}-H_{i} \hat{x}_{i \mid i-1}
\end{array}\right] \\
\delta A & \leftarrow\left[\begin{array}{cc}
-\delta F_{i} & \delta E_{i+1} \\
\delta H_{i} & 0
\end{array}\right] ; \delta b \leftarrow\left[\begin{array}{c}
\delta F_{i} \\
\delta H_{i}
\end{array}\right] \hat{x}_{i \mid i-1} \\
Q & \leftarrow\left[\begin{array}{cc}
P_{i \mid i-1}^{-1} & 0 \\
0 & 0
\end{array}\right] ; W \leftarrow\left[\begin{array}{cc}
Q_{i}^{-1} & 0 \\
0 & R_{i}^{-1}
\end{array}\right] \\
H & \leftarrow\left[\begin{array}{cc}
M_{f, i} & 0 \\
0 & M_{h, i+1}
\end{array}\right] ; N_{b} \leftarrow\left[\begin{array}{c}
N_{f, i} \\
N_{h, i}
\end{array}\right] \hat{x}_{i \mid i-1} . \\
N_{a} & \leftarrow\left[\begin{array}{cc}
-N_{f, i} & N_{e, i+1} \\
N_{h, i} & 0
\end{array}\right] .
\end{aligned}
$$

More details of these proofs can be seen in [13].

\section{REFERENCES}

[1] B. D. O. Anderson and J. B. Moore, Optimal Filtering. Englewood Cliffs, NJ: Prentice-Hall, 1979.

[2] G. J. Bierman, "An application of the square root filter to large-scale linear interconnected systems," IEEE Trans. Automat. Contr., vol. 22, pp. 989-991, 1977.

[3] D. Cobb, "Feedback and pole placement in descriptor variable systems," Int. J. Contr., vol. 33, pp. 1135-1146, 1981.

[4] L. Dai, "Impulsive modes and causality in singular systems," Int. J. Contr., vol. 50, no. 4, pp. 1267-1281, 1989.

[5] M. Darouach and M. Zasadzinski, "State estimation for a class of singular systems," Int. J. Syst. Sci., vol. 23, no. 4, pp. 517-530, 1992.

[6] M. Darouach, M. Zasadzinski, and D. Mehdi, "State estimation of stochastic singular linear systems," Int. J. Syst. Sci., vol. 24, no. 2, pp. 345-354, 1993.

[7] Z. Deng and Y. Liu, "Descriptor Kalman estimators," Int. J. Syst. Sci., vol. 30, no. 11, pp. 1205-1212, 1999.

[8] M. A. Hasan and M. R. Azim-Sadjani, "Noncausal image modeling using descriptor approach," IEEE Trans. Circuits Syst. II, vol. 42, no. 8, pp. 536-540, 1995.

[9] B. Hassibi, A. H. Sayed, and T. Kailath, Indefinite-Quadratic Estimation and Control. New York: Wiley, 1999, SIAM - Studies in Applied and Numerical Mathematics.

[10] A. S. Householder, The Theory of Matrices in Numerical Analysis. Waltham, MA: Blaisdell, 1964.

[11] J. Y. Ishihara, M. H. Terra, and J. C. T. Campos, "Robust Kalman filter for descriptor systems," in Proc. Amer. Contr. Conf., Boston, MA, Jun./ Jul. 2004.

[12] — - "Optimal recursive estimation for discrete-time descriptor systems," Int. J. Syst. Sci., vol. 36, no. 10, pp. 605-615, 2005.

[13] A. Padoan, Jr., "Algoritmos Array Para Sistemas Descritores," (in Portuguese), Master's thesis, Electrical Engineering Department, University of São Paulo at São Carlos, São Carlos, Puerto Rico, 2005.

[14] T. Kailath, A. H. Sayed, and B. Hassibi, Linear Estimation. Upper Saddle River, NJ: Prentice-Hall, 2000.

[15] P. G. Kaminski, A. Bryson, and S. Schmidt, "Discrete square root filtering: A survey of current techniques," IEEE Trans. Automat. Contr., vol. AC-16, pp. 727-736, Dec. 1971.

[16] Y. S. Kim and K. S. Hong, "Federated information mode-matched filters in ACC environment," Int. J. Contr., Automat., Syst., vol. 3, no. 2, pp. 173-182, 2005.

[17] D. V. Luenberger, "Dynamic equations in descriptor form," IEEE Trans. Automat. Contr., vol. 22, pp. 312-321, 1977.

[18] J. K. Mills and A. A. Goldenberg, "Force and position control of manipulators during constrained motion tasks," IEEE Trans. Robot. Automat., vol. 68, pp. 30-46, 1989.

[19] R. W. Newcomb and B. Dziurla, "Some circuits and systems applications of semistate theory," Circuits, Syst., Signal Process., vol. 8, pp. 235-260, 1989.

[20] R. Nikoukhah, S. L. Campbell, and F. Delebecque, "Kalman filtering for general discrete-time linear systems," IEEE Trans. Automat. Contr., vol. 44, pp. 1829-1839, 1999. 
[21] R. Nikoukhah, A. L. Willsky, and B. C. Levy, "Kalman filtering and Riccati equations for descriptor systems," IEEE Trans. Automat. Contr., vol. 37, pp. 1325-1342, 1992.

[22] A. H. Sayed, "A framework for state-space estimation with uncertain models," IEEE Trans. Automat. Contr., vol. AC-46, pp. 998-1013, 2001.

[23] B. L. Stevens and F. L. Lewis, Aircraft Modelling, Dynamics and Control. New York: Wiley, 1991.

[24] M. Verhaegen and P. Van Dooren, "Numerical aspects of different Kalman filter implementations," IEEE Trans. Automat. Contr., vol. 31, pp. 907-917, Oct. 1986.

[25] S. Xu and J. Lam, "Robust stability and stabilization of singular systems: An equivalent characterization," IEEE Trans. Automat. Contr., vol. 49, pp. 568-574, 2004.

[26] S. Xu, J. Lam, and Y. Zhou, " $H_{\infty}$ filtering for singular systems," IEEE Trans. Automat. Contr., vol. 48, pp. 2217-2222, 2003.

[27] H. Zhang, T. Chai, and X. Liu, "A unified approach to optimal state estimation for stochastic singular systems," Automatica, vol. 34, no. 6, pp. 777-781, 1998.

[28] H. S. Zhang, L. Xie, and Y. C. Soh, "Optimal recursive filtering, prediction and smoothing for singular stochastic discrete-time systems," IEEE Trans. Automat. Contr., vol. 44, pp. 2154-2158, 1999.

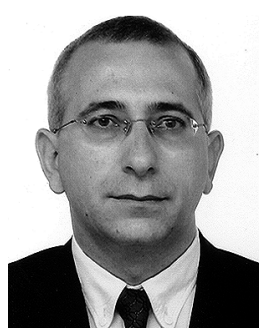

Marco Henrique Terra (M'96) received the Ph.D. degree in electrical engineering from the University of São Paulo (USP) at São Carlos, Brazil, in 1995.

$\mathrm{He}$ is an Associate Professor of Electrical Engineering at USP. His research interests are in the areas of filtering, estimation and control theories, fault detection and isolation problems, and robotics.

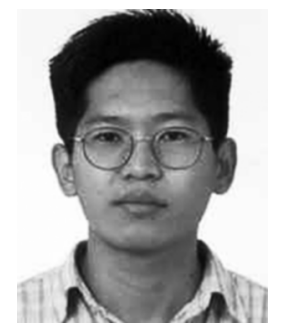

João Yoshiyuki Ishihara received the Ph.D. degree in electrical engineering in 1998 from the University of São Paulo (USP) at São Carlos, Brazil, in 1998.

$\mathrm{He}$ is an Associate Professor of Electrical Engineering at University of Brasilia, Brazil. His current research interests include singular system theory, $\mathrm{H} 2$ control, robust control, and filtering.

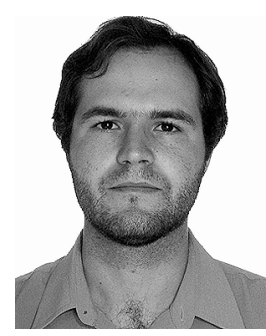

Antonio Carlos Padoan, Jr., received the engineer and master degrees in electrical engineering from the University of São Paulo at São Carlos, Brazil, in 2003 and in 2005 , respectively.

Since 2005 , he has been working with hydraulics, generators, and automation/control of hydraulic power plants at the Alstom Brasil, Ltd. 